\title{
"Organización, fragmentación y posibilidades de cambio: la brecha como vacío fértil"
}

\section{Organization, fragmentation and changes possibilities: the gap as fertile emptness}

Claudia Liliana Perlo ${ }^{1}$

María del Rosario de la Riestra ${ }^{2}$

Leticia del Carmen Costa ${ }^{3}$

\section{Resumen}

En este trabajo buscamos comprender los complejos procesos de aprendizaje colectivo, y explorar y diseñar herramientas que posibiliten desarrollarlo.

De este modo, observamos brechas y fragmentaciones en la red organizativa, que se enquistan en un vacío estéril que impide el flujo y tensión creativa que requieren los procesos de transformación y cambio.

Primeramente se explicitan conceptos, seguido de ejemplos de brechas en torno a las cuales venimos trabajando.

Finalmente concluimos con una síntesis integradora para abrir nuevos horizontes que nos conduzcan a una articulación compleja de dichas brechas.

Palabras-claves: Brecha; Fragmentación; Organización; Vacío fértil.

\section{Abstract}

In this paper we seek to understand the complex processes of collective learning and explore and design tools that enable development.

Thus, we observe gaps and fragmentation in the organizational network, which enclose themselves in a sterile vacuum that prevents the flow and creative tension that processes of transformation and change require.

First of all, concepts are explained, followed by examples of gaps that we have worked on to open new horizons.

Finally, we conclude with an integrative synthesis to open new horizons that will lead to a complex bridging of the gaps.

Key words: Gap; Fragmentation; Organization; Fertile void.

\section{Presentación}

El siguiente trabajo forma parte de nuestra labor de investigación que desde hace más de una década desarrollamos en el Instituto Rosario de Investigaciones en Ciencias de la Educación - IRICE- CONICET, Argentina.

\footnotetext{
Paper received in April 2010 and accepted for publication in June 2010.

${ }^{1}$ Instituto Rosario de Investigación en Ciencias de la Educación (IRICE - CONICET). Doctora en educación. Investigadora del Conicet- Especialista en Aprendizaje y Desarrollo Organizacional. Facilitadora de metodologías actuales para la innovación y transformación de los contextos organizativos. Dirección institucional: 27 de Febrero 210 bis /(2000) Rosario, Argentina. E-mail: perlo@irice-conicet.gov.ar .

${ }^{2}$ Instituto Rosario de Investigación en Ciencias de la Educación (IRICE- CONICET). Doctora en educación. Becaria Post-Doctoral CONICET. Especialista en redes y nuevas tecnologías orientadas al aprendizaje en las organizaciones. Dirección institucional: 27 de Febrero 210 bis /(2000) Rosario, Argentina. E-mail: delariestra@irice-conicet.gov.ar

3 Universidad Autónoma de Entre Ríos. (UADER). Psicóloga. Profesora titular Universidad Autónoma de Entre Ríos. Profesional independiente. Investigadora Adscripta a IRICE- Especialista en conversaciones productivas y enfoques diálogicos en las organizaciones. Su aporte se concentra en dominio personal, y exploración emocional. Dirección institucional: 27 de Febrero 210 bis /(2000) Rosario, Argentina. E-mail: Idelcc@hotmail.com
} 
Con el objeto de pensar la realidad social en términos de construcción y discontinuidad, nos resulta sumamente pertinente presentar un corpus teórico emergente de nuestra investigación y práctica en las organizaciones.

A partir de nuestras investigaciones ${ }^{4}$ observamos brechas y fragmentaciones en la red organizativa, que se enquistan en un vacío estéril o en la visualización desintegrada de las partes. Consideramos que estas modalidades de configuración tornan invisibles el fluir y la tensión dialógica que requieren los procesos de emergencia e innovación en las organizaciones.

En este marco generamos conocimientos para comprender los complejos procesos de aprendizaje colectivo, como así también exploramos y diseñamos herramientas que posibiliten desarrollarlo.

Nuestro enfoque metodológico encuentra fundamento en la perspectiva micropolítica (BALL, 1989; BARDISA RUIZ, 1997; TERRÉN, 2004; BLASÉ; BLASÉ, 1997; LÓPEZ YÁNEZ, 2005) que toma al interaccionismo social (MEAD, 1972) como marco psicosociológico para comprender la acción colectiva en las organizaciones.

En primer lugar explicitaremos el marco conceptual con el que abordamos nuestra tarea. Seguidamente presentaremos ejemplos de brechas y fragmentaciones objeto de análisis de nuestro trabajo de campo.

Posteriormente señalaremos las limitaciones paradigmáticas y culturales que convierten a la brecha en un vacío estéril y a la fragmentación en la asunción de la parte por el todo.

A continuación expondremos nuevos marcos de teóricos para comprender que la configuración de la brecha cómo "agujero improductivo" y la fragmentación cómo "desintegración organizativa", responden al paradigma reduccionista que aún nos atraviesa. Este modo de cartografiar nuestras organizaciones es una condición profundamente limitante en nuestra capacidad operativa para el aprendizaje y el cambio organizacional.

El enfoque complejo (MORIN, 1995) que enmarca nuestro trabajo sostenido desde una visión sistémica de las organizaciones (BATESON, 1991) nos permite abordar finalmente la brecha como vacío fértil y la posibilidad de habitarla en tanto pura potencialidad que provocan los procesos emergentes y el flujo creativo de la realidad concebida como multidimensional.

\section{Marco conceptual}

La organización social influye en la medida en que configura situaciones en cuyo seno actúan los individuos, y en la medida en la que proporciona unos conjuntos estables de símbolos que los individuos utilizan al interpretar las situaciones.

..."Desde el interaccionismo simbólico, la organización es un marco en cuyo interior tiene lugar la acción social pero no constituye el factor determinante de la misma.

Dicha organización y las modificaciones que en su interior se producen son el producto de la actividad de las unidades obrantes"... (BLUMER, 1969 apud GALTIERI, 1992, p.17)

A partir de aquí, se hace impensable sostener que las organizaciones son exclusivamente el resultado de la acción externa de "otros" diferentes a los miembros que la componen, desconociendo la participación individual en la acción organizacional.

La organización es un marco en cuyo seno llevan a cabo sus acciones las personas (para Blumer "unidades obrantes") no lo hacen solo en función de la cultura, la estructura social, etc., sino también en función de las situaciones concretas que se les presentan.

4 "La gestión del cambio a través del aprendizaje de competencias formativas y organizativas" (2004- 2007) y "Aprendizaje y desarrollo organizacional: las concepciones del poder y su incidencia en los procesos de cambio colectivo". (2008-2010) Área de Aprendizaje y Desarrollo Organizacional - IRICE- Consejo Nacional de Investigaciones Científicas y Técnicas- CONICET - Argentina 
Este enfoque, como señala Alcover (2003 apud ESTRAMIANA, 2003), corre el significado de organización desde el de "efecto de organizar u organizarse, (cosificación) al de acción de organizar u organizarse (participación)".

Las organizaciones no son sólo "algo dado", mucho menos un fenómeno natural que el hombre aún no ha podido dominar, una pesada carga de la naturaleza. Sin ignorar la fuerte influencia de las estructuras políticas e ideológicas (instituciones) las organizaciones son también, en parte, aquello que sus miembros han podido conversar, negociar, acordar y construir.

Del mismo modo que ya no se concibe el sometimiento de los individuos a la sociedad como una realidad externa a ellos, tampoco se concibe a las organizaciones como entidades por encima de los individuos y enfrentadas a estos últimos. De lo que sí se trata es de mostrar las influencias recíprocas, interacciones e influencias mutuas entre individuo y organización, del mismo modo que la sociología da cuenta del interaccionismo entre individuo y sociedad. Después de todo, las organizaciones no son otra cosa que el escenario actual donde se desarrolla la vida social. (DRUCKER, 1950)

La red que resulta el colectivo u organización, se constituye en el tejido relacional donde los individuos están presentes.

Las formas reticulares o rizomáticas son las que posibilitarán una comprensión holística del proceso organizacional.

Ahora bien, en tales organizaciones observamos brechas y fragmentaciones que pueden ser consideradas como un "vacío estéril" (PEARLS, 1976) que anula las posibilidades de producir formas nuevas de sentir, pensar y actuar colectivamente.

Es momento de diferenciar, como ya lo anticipamos en la presentación del trabajo, brecha de fragmentación organizativa. Cuando hablamos de fragmentación, aludimos a una concepción atomizada de la organización que impide percibir la naturaleza sistémica y holográmica de la misma. En este sentido la organización es entendida como suma de partes (fragmentos) aislados, referida en la tradicional definición de organización entendida como "conjunto de personas reunidas para la consecución de un fin común". No se trata pues de una re-unión de partes sino de un TODO conformado por subtotalidades (BÖHM, 1980) holográmicas que producen un comportamiento entramado y colectivo. La naturaleza del SER se encuentra en el TODO.

En cambio, percibir la brecha organizativa, implica reconocer una red agujereada, un vacío en la trama por pérdida o ausencia de significado entre los actores entramados. Este agujero cumple la potente función del vórtice (BRIGG; PEATT, 1998) capaz de conducirnos por las profundidades del torrente caudaloso de la totalidad, multidimensionalidad que constituye la realidad cuántica.

Para Argyris y Schön (1978) la brecha o ruptura (gaps/gulf) se manifiesta en la diferencia entre la teoría en uso y la teoría declarada (ARGYRIS; SCHON, 1974). Los autores utilizan estas categorías para distinguir aquello que las personas enuncian en las organizaciones tanto como políticas adoptadas o como prácticas colectivas deseadas y aceptadas (TD); de aquellos supuestos que las personas sostienen de manera subyacente en la acción y que efectivamente llevan a cabo (TU).

Desde la ciencia de la acción explorar el campo de significados latentes entre las teorías en uso y las teorías declaradas implica trascender de un aprendizaje de lazo simple hacia un aprendizaje de lazo doble. El aprendizaje de lazo simple "se produce cuando los miembros de una organización responden cooperativamente a los cambios en el contexto interno y externo de ésta, detectando errores que pueden corregir, evaluando y generalizando los resultados mientras mantienen los rasgos centrales de la teoría en uso. Este tipo de aprendizaje permite mantener sin cambio las normas de la organización". Mientras que el aprendizaje de lazo doble "es capaz de cuestionarse a si mismo como sistema de aprendizaje, es un proceso de indagación acerca de la detección y corrección de errores basados en la incongruencia entre las teorías declaradas y las teorías en uso. Este es un proceso dinámico y de cambio, tendiente a la transformación del status quo de la organización" (PERLO, 2006a:148) 
Asimismo se considera que la brecha entre ambas teorías, no es en principio estéril en tanto mantiene ambas teorías vinculadas y brinda la posibilidad de crear una dinámica de exploración dando lugar a la reflexión y al diálogo.

En cuanto consideramos que las conversaciones conforman las organizaciones (Flores 1994), las brechas que encontramos en ellas constituyen los intersticios generativos de los nuevos significados para transformar la acción.

La ciencia de la computación conceptualiza esta brecha como ausencia de información.

En tal sentido el proceso de facilitación debe focalizarse en torno a tomar conciencia sobre dicha brecha y trabajar en ella buscando reconstruir la fragmentación de la información y ampliar el campo de significado que la misma implica para los actores involucrados.

En convergencia con lo que venimos desarrollando, el enfoque Gestáltico, considera que dicho vacío estéril puede transformarse en un "vacío fértil". Nuestra práctica nos ha mostrado la alta potencialidad de cambio que brinda la posibilidad de ubicarnos en la brecha que produce el vacío de "no ser" lo uno u lo otro. La tarea consiste en sostenerse en dicho lugar e indagar creativamente, en torno a la multidimensionalidad que brinda la realidad como totalidad compleja. El vacío podrá transformarse en fértil en la medida que podamos reconocer que cada uno de nosotros somos seres esencialmente creativos.

Es interesante señalar que desde la física la brecha de energía es la "no existencia" de electrones y a su vez es lo que permite la liberación del electrón de la órbita del núcleo para moverse libremente por el sólido.

"La clave para la actividad creativa reside en la autoorganización de los materiales disponibles. Para los humanos esto significa que debemos crear con el material de nuestras propias vidas" (BRIGGS; PEAT, 1999:21)

La brecha también puede ser considerada fuente de "tensión creativa" (SENGE, 1992, p.193). La brecha entre la visión propia de la realidad y la realidad misma es una fuente de energía. "Sino habría brecha, no habría necesidad de una acción para moverse hacia la visión. La brecha es fuente de energía creativa. Llamamos a esta brecha tensión creativa”. (SENGE, 1992, p.193)

La tensión tiende a la resolución de la misma o a la liberación. "La tensión creativa es la fuerza que entra en juego en cuanto reconocemos una visión que está reñida con la realidad actual”. (SENGE, 1992,p.193)

\section{Las brechas en la red organizativa}

Las brechas que observamos en las organizaciones se asientan en la tensión reactiva entre:

- Las teorías declaradas --- las teorías en uso (ARGYRIS, 1999)

Tanto en la vida personal como de las organizaciones se observan múltiples manifestaciones de la brecha existente entre las teorías declaradas y las teorías en uso que sostienen las personas.

Innumerables ejemplos observamos y recogemos en las organizaciones. Muestra de ellos es la gerencia que se encuentra altamente comprometida con las normas de calidad y de hecho aprueban los requisitos de Normas ISO. Sin embargo, ante la presencia de los auditores se muestra "solo aquello con lo que se pudo cumplir" Ante lo cual los responsables del proceso justifican que no pueden cumplir con todo lo que prevé la norma, ya que el costo es muy elevado.

Asimismo en las organizaciones escolares se expresa que los contenidos deben ser enseñados como problemas de las ciencias y del contexto social donde éstos emergen, paralelamente la práctica curricular se impone organizando dichos contenidos en torno asignatura: matemática, lenguaje, historia.

Del mismo modo que muchas empresas declaran que respetan el medio ambiente, aunque en su uso se desechan a los afluentes los desperdicios productivos ya que nadie controla y además todos lo hacen igual. 
Es común escuchar en las organizaciones productivas que su metodología está basadas en normas y procedimientos estándar, aunque es bien sabido que en la práctica generalmente el operador desconoce tal procedimiento ya que se vale por su experiencia.

Los profesionales de la educación sostienen que cada alumno tiene un ritmo de aprendizaje diferente y esta diversidad no solo debe ser tenida en cuenta, sino además vista como una ventaja para el enriquecimiento del aprendizaje en la clase. Cada alumno es un ser único e irrepetible y esto constituye un valor en el proceso de aprendizaje. En la práctica áulica se considera que los alumnos que no se ajustan al ritmo común de la clase tienen una dificultad, la heterogeneidad que presentan éstos alumnos es vista como un problema a resolver.

Podríamos continuar con numerosas situaciones que denotan un enquistamiento en estas brechas no exploradas, que se encuentran allí a la espera de un observador atento e inquieto que recupere su significado y permita que de la fertilidad de dicho vacío florezca un cambio transformativo.

- La persona--------- El puesto

En las organizaciones observamos una fuerte tensión entre considerar el puesto como determinante de la persona o viceversa. Comúnmente las personas son desvinculadas de ciertos puestos en la medida que despliegan sus talentos que exceden lo esperado para el mismo.

Asimismo observamos la fragmentación tanto en el discurso como en la práctica, de lo que significa vincularse, según se entable una relación personal con "Juan Gómez" o "Carlos Gonzáles" o una relación laboral referida al puesto, con "el gerente", "el empleado, el directivo o el jefe".

Asimismo ante una búsqueda de personal, generalmente se seleccionan las personas según su perfil "encaje" con el puesto buscado.

La tensión esta planteada en tanto los puestos, los roles siempre serán espacios determinados y limitados por tareas y funciones a cumplir, mientras que las personas tienen además de características particulares más o menos estables un potencial de desarrollo creativo inmensurable vinculado e interconectado a una red psicosocial y organizativa de la cual emerge inhibiéndose o transformándose de acuerdo a las posibilidades o limitaciones que el tejido genere.

- Los intereses personales------los intereses colectivos

En primer lugar la alusión a "intereses personales" tiene una connotación negativa en el contexto organizativo, como si fuera posible deshacerse de ellos. Los intereses colectivos, se perciben separadamente de estos primeros y son colocados en un lugar meritorio y laudatorio, privativo de aquellos que "se entregan" a la organización resignando "sus intereses personales" en pro de un "bien común y colectivo". En las empresas la expresión "ponerse la camiseta" refiere a este comportamiento organizativo que denota además orgullo y gratitud por el lugar que en la misma se le ha concedido.

Sin embargo esta percepción disyuntora de los intereses personales y los intereses colectivos provoca en muchas ocasiones el crecimiento individual de que no conduce generalmente al desarrollo del colectivo o bien un crecimiento corporativo que no implica desarrollo de las personas.

Consideramos que el crecimiento colectivo puede generar crecimiento individual entre quienes lo integran y viceversa en tanto mediante un proceso de aprendizaje organizacional pueda reconocer la trama implícita que transforme esta brecha.

- Los de arriba (ustedes) -------- los de debajo (nosotros).

La concepción mecánica de la realidad social que se desprende de la metáfora de la organización como una pirámide, fuertemente arraigada en nuestras práctica sociales, conduce a nuestro ojo observador a capturar un espacio organizacional configurado entre "el arriba y el abajo" impidiéndonos advertir la fluidez de los procesos en los que estamos involucrados. De este modo en las organizaciones escuchamos más comúnmente entre los empleados considerados de menor jerarquía, un discurso que se dirime entre "nosotros y ustedes". El 
"nosotros" suele aludir a la base de la pirámide y "ustedes" a la cúspide de ésta. Esta visión fragmentaria impide "pensar juntos" la acción colectiva en la que cada uno individualmente (hilos de la trama) participa de manera incoherente y asistémica en la construcción de una realidad organizacional que paradójicamente desearía que cambie.

La percepción de la organización a través de la metáfora de la pirámide nos condena al desencuentro con el otro, a la imposibilidad de apreciar la totalidad. Contrariamente la percepción reticular de la organización nos conecta con el placer del encuentro con los otros y enriquecimiento en su diversidad.

- El SI incondicional al cliente ------------- NO como ineficiencia del servicio

En las organizaciones, en especial las abocadas a los servicios de gestión privada se impone la ley del mercado en la cual "El cliente siempre tiene la razón", el NO al cliente es un indicador de "mal servicio" o "baja calidad en la atención".

El NO expresado en la primera línea es tomado por el cliente como ineficiencia y mala calidad en el servicio, el que corrobora cuando su demanda se resuelve en cualquiera de las líneas superiores, asimismo comparte esta perspectiva con sus colegas generando una visión colectiva de "mal servicio" por parte de la empresa.

En la primera línea los empleados se sienten exigidos a responder siempre SI aceptando resolver problemas con o sin solución de manera inmediata, para evitar la descalificación del servicio. El Si hacia afuera del mostrador que permite conformar y tranquilizar al cliente, se vuelve disconformidad y malestar hacia el interior del mostrador, que genera presión, tensión, stress, malhumor, violencia, restando calidad al trabajo interno del equipo, produciendo mal- estar y por ende bajando la eficiencia en la prestación del servicio, ratificación que podría volver a comprobar de todos modos el cliente.

El SI y el NO en tanto dos respuestas irreconciliables se enquistan en una brecha estéril que impide pensar creativamente otras alternativas que pudieran encontrarse en la realidad concebida como multidimensional.

Asimismo consideramos que esta subordinación al otro por parte de los empleados que se encuentran en la primera línea de atención (por ellos denominada trinchera) reproduce la vieja relación amo y esclavo. En esta relación los actores sociales en un estado de ánimo de despontenciación (ASCORRA, 2009) producto de acatar y no participar en la reglas, reprime su acción y lo que es peor aún su natural potencia.

- La vida personal---- la vida laboral

Desde la metáfora de la máquina, la vida personal, los problemas personales, la familia, en definitiva las emociones quedan escindidas de la vida laboral. En el discurso de los actores: "Los problemas personales deben quedar en la puerta del trabajo, y los problemas del trabajo en la puerta de la casa".

Asimismo recogemos expresiones tales como "Yo en mi casa me relajo y puedo disfrutar, luego de llegar del trabajo donde estoy todo el tiempo tensionado y uno no se puede relajar ni un minuto". Las mismas que denotan un control externo de las emociones determinado por el contexto organizativo donde se asume el contexto laboral como lugar de presión y el contexto personal/fliar en el cual puede haber lugar para la distensión. Lo cierto es que las personas pasan demasiadas horas en las organizaciones, constituyéndose éstas últimas en parte central de su "vida personal", por lo cual $e$ l enquistamiento en esta brecha constituye una seria dificultad para comprender la conexión del individuo con la realidad indivisible a la que pertenecemos. La vida laboral y la vida personal es otra fragmentación de la modernidad occidental que impide una visión sistémica de la realidad.

\section{Limitaciones para habitar la brecha}

Las brechas en sí mismas no constituyen una dificultad para aprender un posible diferente. Desde el inicio, las dificultades aparecen cuando no incluimos la posibilidad de "puntos ciegos" en nuestros modos de co- habitar territorios existenciales. 
El no reconocimiento de nuestra limitada percepción humana se constituye en un factor predisponente para alimentar las restricciones a la hora de habitar las brechas debido a que, somos incapaces de percibir, sentir, emocionar y pensar todo lo que acontece en nuestra cotidianeidad. Reconocer que co-habitamos nuestros mundos sociales cartografiando mapas de infinitos territorios, implica aceptar la limitación de nuestros registros, no admitir esta condición obstaculiza el acercamiento con aspectos negados, confinados a la sombra. En consecuencia se restringe la posibilidad de "expandir la identidad; restableciendo el contacto con capacidades ocultas para asimilar los mensajes procedentes del lado oscuro”. (DOMATO, 2007)

Tal cuestión se agrava cuando no tomamos conciencia hasta qué punto nos encontramos atrapados en marcos culturales, donde prevalecen ciertos modos de organizar nuestras vivencias y experiencias priorizando algunas percepciones por sobre otras. Esta configuración de la realidad vivida se realiza en función de ciertas normativas sociales y leyes instituídas finalmente avalados bajo irrefutables parámetros de la ciencia normativa. Ejemplo de esto, es la prevalencía de la visión fragmentada con la cual aún hoy observamos los procesos sociales que nos constituyen. Positivamente y con una mirada reduccionista diseñamos soluciones a los problemas que presenta nuestra sociedad organizada, abocados con empeño a la construcción de escenarios que propician la frustración, y el malestar colectivo avivando la llama del dolor. De este modo solemos recaer en profecías autocumplidas, entrenando con ahínco nuevas habilidades incompetentes para eludir la brecha sin poder reconocer los puntos ciegos que alimentan el infortunio. Desde esta perspectiva, las habilidades incompetentes se nutren retroactivamente de patologías relacionales. David Bhom (1980) reconoce al menos cuatro, que dificultan la generación de espacios inclusivos entre los sujetos y obstaculizan el "pensar juntos" un posible diferente. Ellos son la abstracción, la certeza, la idolatría y la violencia.

La abstracción implica un ensimismamiento en el propio modelo mental, la certeza refiere a la ausencia de suspensión, la idolatría indica que "me escucho solo a mi mismo" y finalmente la violencia significa el no respeto y la agresión al otro.

En este sentido Abstracción, Certeza, Idolatría y Violencia se constituyen en factores precipitantes de las limitaciones para habitar la brecha que agudizan el enquistamiento en la visión fragmentada de las organizaciones junto la deserción de pensar juntos un posible diferente.

Los escenarios para la frustración se instalan poderosamente cuando no aceptamos la posibilidad de vivir una invitación. Aquella que nos convida a aprender y diseñar nuestros mapas cotidianos con múltiples maneras de enfocar, donde la idea de pliegue y despliegue de posibilidades ocupa la observación. Esta se enmarca desde una innovadora estética de la complejidad donde no hay rumbo lineal, ni fijo sino configuraciones en permanente fluir y emergencia.

La posibilidad de configurar implica que accedemos a distintas perspectivas, incluyendo cortes transversales y movimientos de barrido, elección de escala y modo de interacción. En suma, se trata de un dispositivo que nos da la posibilidad de construir un estilo de indagación caracterizado por la exploración. (NAJMANOVICH, 2008)

Para tal fin no basta con trabajar en torno a la apertura de nuestro registro perceptual en su totalidad, la apuesta implica un más allá de la mera ampliación de foco .Se impone aquí la invitación a configurar desde una estética diferente atravesada por una epistemología holística y vital con una lógica no lineal enriquecida por una creativa forma de vivir en este universo donde es preciso estar atentos en "awareness", para crear nuevos territorios existenciales.

Pareciera que nuestra propia fisiología nos indica por si misma el orden de importancia que tienen nuestros órganos sensoriales para percibir el mundo que nos rodea. Contamos con un par de oídos, un par de ojos y una sola boca, los ojos y la boca pueden abrirse o cerrarse a nuestra voluntad, mientras que nuestros oídos siempre están allí prestos para escuchar. Deducimos entonces que nuestra naturaleza fisiológica nos enseña que la escucha es prioritaria por sobre nuestra vista, capacidad para observar y ambas por sobre nuestra boca, capacidad para emitir nuestro pensamiento a posteriori de una escucha atenta y una observación profunda. 
Esta observación profunda requiere del desarrollo de la "percepción sensible" aquella que nos permite articular una mente que siente, junto a un corazón que piensa relacionalmente.

Se trata de centrar la atención en las emociones que produce dicha observación y no en las ideas, prejuicios y conocimiento previos que tenemos de esa situación, ello permite dar nuevo sentido a lo que observamos y aún más integrando lo que esa situación y/o sujeto nos quiere comunicar. Se trata de ampliar la percepción interna de la realidad. Al respecto señala el poeta William Blake: "Si se abrieran las puertas de la percepción, veríamos las cosas como realmente son: infinitas."

\section{Nuevos marcos de comprensión para el análisis de la fragmentación de la red}

Para transformar el vacío estéril en vacío fértil ampliar nuestra percepción será una tarea ineludible. En tal sentido, cambiar nuestra concepción ontológica de ser y realidad, desde una perspectiva cuántica transformar nuestra visión individual, mecánica, disyuntiva y sustantiva de la realidad organizacional, por una visión compleja, reticular, fluida y holográmica de la misma, para lo cual debemos comprender la realidad y al ser como devenir y al individuo como subtotalidad organizacional.

\section{Realidad, ser y devenir}

Durante el siglo XX, la teoría de la relatividad (EINSTEIN, 1905) la mecánica cuántica (Bohr y la concepción del átomo) junto a los descubrimientos de Heisenberg (principio de incertidumbre) y a los aportes Schröringer (función ondulatoria), propiciaron una revolución paradigmática que cambiaría radicalmente nuestra concepción de la realidad.

"El principio de indeterminación de Heisenberg mostraba que las propiedades reales de los objetos ya no se podían separar del acto de la medición y por ende del observador mismo" (BRIGGS; PEAT 1999:54)

La totalidad de lo que existe en el universo está conformado por información y energía. Estas se constituyen en manifestaciones diferentes de la misma cosa por lo tanto "Somos expresiones diferentes de energía e información." (EINSTEIN) ..."La luz y otras formas de energía tienen doble personalidad y a veces se comportan como ondas y a veces como partículas" (BRIGGS; PEAT, 1999:43)

En este sentido la realidad no es definitiva, no es regular, no es inmutable, en la naturaleza encontramos más que cosas que existen, cosas a existir, que "son" (existen) en la medida que las configuramos a través de la observación.

Desde estas perspectiva el observador es lo observado, podemos reconocer dos dimensiones de la realidad, lo que está siendo (orden explícito, BÖHM, 1980) y lo que puede ser (orden implícito, BÖHM, 1980). La naturaleza es discontinua, lo que separa "lo que está siendo" de lo que "puede ser" es nuestra observación, la que no se orienta a través de una trayectoria de causas y efectos, sino de saltos probabilísticas y correlaciones.

El investigador Niels Bohr postula que es la conciencia, la responsable de la mutación onda-partícula, por lo tanto la realidad "es circunscripta" a un determinado contexto para un grupo determinado de observadores y existe en una potencialidad pura (universo plegado, BÖHM, 1980) y se constituye como tal (universo desplegado, BÖHM, 1980) en tanto la observamos (creamos).

Provocados por este marco de comprensión de la realidad, podemos registrar por lo menos dos ámbitos de existencia: el físico, el que hemos circunscripto, fijado, a través de nuestra observación utilizando nuestros sentidos y el virtual, o no circunscripto, es el ámbito de lo potencial donde algo puede ser partícula y onda simultáneamente. La observación será la que convierte a una o a otra en realidad.

"El observador y los observado parecen influirse mutuamente, el científico es como un remolino tratando de estudiar el flujo del agua" (BRIGGS; PEAT, 1999, p.35) 
Los avances empíricos de la física cuántica a lo largo de todo el siglo pasado han demostrado la existencia de una realidad no circunscripta que fluye más al modo del río que del remolino (realidad circunscripta). Esta concepción de la realidad donde ser (remolino) y devenir (río) se entraman, constituye un afluente que nutre un proceso de aprendizaje capaz de producir un cambio transformador en los sistemas.

La construcción de la realidad se entiende como la coordinación del flujo inevitable de movimientos de pliegue y despliegue que van configurando diversos órdenes. En lo plegado (orden implícito), las cosas fluyen unas con otras en la totalidad. En lo desplegado (orden explícito) se configuran subtotalidades relativamente autónomas de la red integral.

Lo que aparece ante nuestra mirada como discontinuo y azaroso, en realidad constituye diferentes órdenes de pliegue y despliegue de los fenómenos. Entonces debajo de todo orden explícito, existe un orden implícito. Podemos aventurar desde aquí qué la partícula ya no es una cosa, sino despliegues sucesivos, al mismo tiempo que es siempre lo mismo ya que se encuentra plegada en todo lo demás. En consecuencia lo que se transforma es nuestra comprensión de lo implícito que produce diferentes grados de explicitación de las "subtotalidades relativamente autónomas".

La presencia relevante del orden explícito que ha posibilitado al hombre innumerables avances en la exploración de los subtotales (ciencia y tecnología), a su vez lo ha inhibido en la compresión del desapercibido orden implícito, donde lo no desplegado aún, le brinda infinitas posibilidades para transformarse y transformar la realidad única en la que vive a partir del menú que ofrece la realidad multidimensional.

A partir de este recorrido podemos inferir que, para producir un cambio de sistema será necesaria una coordinación entre las subtotalidades y la totalidad que no obstruyan la fluidez del movimiento. El aprendizaje emerge como un proceso de despliegue hacia lo desconocido en la totalidad.

Debemos aprender del movimiento, de lo que fluye inevitablemente. El universo se mueve, debemos aprender a acompañar el movimiento sin fuerza, sin presión, percibiendo nuestra interacción con la totalidad, moviéndonos sincrónicamente con lo que acontece, de manera similar a "como el esquiador que se desliza según la curvatura del terreno". (MATURANA, 2007)

No interponernos, ni interrumpir al movimiento, solo permitiéndolo; dándole paso somos con el movimiento, no somos más que él, del mismo modo que la ola se mueve como parte y todo con el océano.

La comprensión de esta concepción posibilita la apertura necesaria de las unidades autopoiéticas para que fluya el todo hacia la subtotalidad generando una disposición interna para que un cambio de tipo transformativo acontezca.

\section{El individuo como subtotalidad organizacional}

En nuestras investigaciones advertimos que la concepción mecánica de los fenómenos sociales impide advertir la fluidez de los procesos en los que estamos involucrados. Si bien nuestra labor se focalizó en la exploración de la dinámica relacional individuo - organización fue indispensable realizar una profunda reflexión entorno a la constitución del fenómeno colectivo en la dimensión social. En este marco los aportes de la teoría de la complejidad (MORIN, 1995) y las teorías de la totalidad (BÖHM, 1980) nos condujeron hacia la relación parte-todo.

Configurar individuos y organizaciones separadamente es una abstracción de "subtotales" que produce nuestro pensamiento y que a los fines de comprender la acción colectiva es inadecuada. Esta proposición se fundamenta en los trabajos de Böhm (1980) en los cuales propone que en el "orden implícito o implicado del universo", los fenómenos no están constituidos por partes sino que las "cosas" se contienen mutuamente. Ahora entonces, podemos inferir que la organización (colectivo) es el resultado del entramado relacional que de manera intencional o no establecen los puntos/hilos (individuos) que la constituyen. 
El mundo aparece entonces como un complicado tejido de acontecimientos, en el que conexiones de distinta índole alternan o se superponen o se combinan, determinando casi la textura del conjunto. (CAPRA, 1996).

Del mismo modo que un "tejido junto" (complexus) (MORIN, 1995) no es sin un orden determinado de los hilos que lo componen, una organización jamás será únicamente un organigrama o estructura en la mente de algunos individuos o en un papel.

La organización es un enmarañamiento de procesos. Estos constituyen una red móvil que produce relaciones entre diferentes puntos, (hilos), las organizaciones como tales son producto y productoras de dicha red. Una organización es un holomovimiento, (BÖHM, 1980) entre trama e hilos, incluyendo en éste, en todo caso, su organigrama.

De este modo las partes y los fragmentos existen en tanto "autonomías relativas".

Del mismo modo que una ola constituye una entidad "separada" del océano, "relativamente", en tanto no sería tal fuera de éste. Por lo que las cosas, como también nosotros mismos, constituimos "subtotalidades relativamente autónomas" del movimiento fluido de la totalidad.

Desde el enfoque de los nuevos paradigmas, procesos fluidos y movimientos constituyen la trama organizativa. En este sentido la organización emerge como totalidad qué no admite arriba y abajo, según captura nuestro ojo conceptualizador (estructura piramidal) sino que constituye una red dentro de un sistema.

Este postulado puede parecer sencillo y obvio, sin embargo continuamos diseñando estructuras organizativas escindidas de los procesos sociales configurados y observando fragmentariamente las tramas, el tejido por un lado y los hilos por otro, no pudiendo reconocer nuestra individualidad entramada.

Nuestra cultura occidental tiene una fuerte impronta de la conciencia explícita, estamos entrenados para abstraer subtotales, que muy comúnmente tienen poco de relativos y mucho de estables.

La exacerbación de las partes, "subtotales", el énfasis en el "yo circunscripto", nos imposibilita observar el colectivo como parte de nosotros mismos, comprendiendo que somos para sí, a la vez que somos para otros. (MEAD, 1972; COHEN, 2005).

El proceso de individuación a través de la construcción de la identidad nos conduce a una circunscripción estricta y rígida del "yo" y "los otros", que deja poco margen para advertir el orden implícito subyacente que tiene la existencia individual y su naturaleza holográfica que le devuelve el reflejo de la totalidad. Esto produce una seria dificultad para comprender lo colectivo como un "nosotros entramado".

Participamos de una identidad mayor que constituye "la trama de la vida". (CAPRA, 1996).Teniendo en cuenta a Böhm (1980) que cada uno de nosotros somos subtotalidades, debemos comprender al otro no como amenaza, sino como integración a la totalidad.

\section{Del vacío estéril al vacío fértil. La brecha como pura potencialidad creativa.}

Para este equipo la brecha se configura cómo pura potencialidad. Esta es una condición indispensable para la facilitación de procesos emergentes, junto a la confianza en la abundancia de recursos disponibles y aun no reconocidos para el surgimiento del flujo creativo.

Entender la brecha como pura potencialidad requiere identificar los procesos emergentes y el flujo creativo que constituye la realidad entendida como multidimensional, tal como concebimos a partir de los nuevos modelos de comprensión anteriormente expuestos provenientes desde diferentes marcos disciplinarios que hoy conforman el pensamiento complejo.

En este sentido emergencia y creatividad constituyen dos puntos de apalancamiento (SENGE, 1992) para sacar provecho de la potencialidad y fertilidad que implica el vacío. 
Los hechos, sucesos, acontecimientos, la realidad misma no posee una existencia inmutable independiente de nuestra observación. La autentica naturaleza de las cosas no está dada por la solidez de los objetos sino por el vacío confinado a la interpretación del mundo circunscripto por lo observado. De allí su fertilidad.

Asimismo, hablamos de puntos de apalancamiento porque "La clave del pensamiento sistémico es la palanca: hallar el punto donde los actos y modificaciones en estructuras pueden conducir a mejoras significativas y duraderas" (SENGE, 1992:148)

El apalancamiento es un principio universal se ha encontrado múltiples aplicaciones en campos como la mecánica, las economía y la gestión, y también podemos encontrar su utilidad para el aprendizaje y desarrollo colectivo.

El punto de apalancamiento de los sistemas reales no es obvio para la mayoría de los actores. Ellos no ven la estructura subyacente de los actos. Lamentablemente cuanto más movemos las palancas tradicionales, mas resistencia se causa. Generalmente intentamos una compensación mediante mayor esfuerzo, y hacemos más de lo mismo. El arte del pensamiento sistémico consiste en ser capaz de reconocer estructuras sutiles de complejidad creciente (dinámica). El punto de apalancamiento se encuentra en el rizo compensador, no el rizo estabilizador.

Los objetos y su movimiento son emergentes, son totalmente reales sólo que su realidad es diferente a la clásica (NAJMANOVICH, 2008). Al mencionar movimientos y procesos emergentes hacemos referencia a las propiedades y cualidades de un sistema que no son reductibles a las propiedades o procesos de sus partes constituyentes. Estas categorías se encuentran en estrecha relación con los conceptos de supervivencia y autoecoorganización, constituyen un quiebre paradigmático en relación al reduccionismo y al dualismo. En este sentido podemos inferir que hay una dinámica de desarrollo cognoscible pero no predecible. Se ha roto la ligadura entre "determinismo y predicción", el sistema es "tratable" pero no "manipulable", está siempre al borde del caos.

Una visión creativa de la realidad implica servirse de la superabundancia de la multiplicidad de posibilidades que constituye la realidad. Requiere sumergirse en lo extraordinario.

Todos somos creadores y creativos, como expresa Toro Araneda (2010): "el concepto elitista, que separa a los creadores de las personas comunes, no representa sino uno de los tantos prejuicios culturales que, en el fondo, refuerzan la trágica disociación del hombre y su obra”.

Debemos recuperar el desarrollo natural de nuestra función creativa. Análogo a la naturaleza, esta función innata posibilita fructificar en términos de desarrollo humano.

En las organizaciones comúnmente se aprende a disociar lo afectivo de la tarea, a mecanizar el vivir obstruyendo la expresión del natural impulso creador.

En las organizaciones actuales, entendidas como máquinas, se requiere de valor y coraje para expresar el impulso creativo.

Para ingresar en el proceso creativo hay que desplazarse de lo simple a lo complejo, de lo diferenciado a lo indiferenciado, este desplazamiento nos permitirá el encuentro con la diversidad de posibilidades que ofrece la realidad múltiple.

Asimismo como señala Toro Araneda (2010), el desafío estará en la humildad para abandonar nuestras múltiples opciones que brinda el universo infinito y elegir la posibilidad más adecuada para la transformación de esa realidad.

En este sentido el "recreo" para "abrir el juego", para re- crearnos y re- crear nuestro hacer, es más adecuado que la clase que es dictada, que en-seña y que marca con lo que ya está sustantivado. 
Se trata de explorar los vacíos, dejar emerger lo que está sumergido, ampliar horizontes, re-crear mundos de experiencia, crear nuevos vínculos, debilitar viejos lazos.

\section{Del cómo habitar la brecha}

La sustantivación de los fenómenos, ha convertido el pensador en cartógrafo, a pesar que la física cuántica, ya desde principios del siglo XX vienen poniendo énfasis más en las interacciones que en las fuerzas y más en los campos de energía que en las partículas. (SERNA ARANGO, 2009).

Para utilizar el potencial creativo de las brechas, necesitamos contar con más pensadores que cartógrafos. Necesitamos aprender a mirar con otros ojos y a registrar con una mente que siente y un corazón que piensa.

Asimismo necesitamos reconocernos co-creador responsables de la realidad organizacional en la que participamos. (DAVILA YÁNEZ; MATURANA, 2007; PAYMAL, 2008)

Reconocer la capacidad de poder autodeterminarnos -autopoiésis- nos brinda una relación más amplia con la totalidad y sus infinitas posibilidades (expansión) y consecuentemente nos vuelve más inestables. Lo que conlleva implícito el potencial de una mayor disposición interna (flexibilidad) a un cambio de sistema.

Lo que deberíamos aprender, valiéndonos de la capacidad autónoma que nos proporciona nuestra organización autopoiética, es la oportunidad permanente que nos ofrece el holomovimiento (totalidad) para desplegar nuestro potencial (poder) creador. Este tipo de aprendizaje puede orientarnos hacia un cambio transformador. Cuando hablamos de co-creacion, esta implícito el Otro, con quien necesitamos redefinir nuestra relación. El Otro debe dejar de ser entendido como una amenaza para ser comprendido como encuentro con la totalidad. O bien como señala Enrique Fernández Longo (1997), como mi patrimonio.

Debemos considerar la posibilidad de elegir entre la tensión reactiva frente el otro/estructura, o la tensión creativa con el otro para cogestionar una nueva estructura.

Se requiere pasar de la metáfora de la pirámide a la metáfora de la red para comprender la acción colectiva en tanto ensambles autoorganizados, que deben explicitarse para conocer y revisar el vínculo que se establece cuando participamos en la trama. En este sentido, la relación con el otro se encuentra configurada en la comunicación. La comunicación es la operación básica de la sociedad (LUHMANN, 1987). En trabajos anteriores (COSTA; PERLO; DE LA RIESTRA, 2005, 2008) hemos profundizado en el proceso dialógico como herramienta para la construcción de la mente colectiva en el contexto organizativo.

Al respecto, actualmente coincidimos con Moreno, cuando expresa:

"En la modernidad el diálogo no es pensable sino como acuerdo, consenso o aceptación. En el pueblo el diálogo se-vive-en la comprensión-afectividad aunque no se produzcan acuerdos. Lo afectivo conversa y dispone. El diálogo es comunicación: la comunicación en el pueblo es afectividad relacionante y relación afectivamente en que se conoce y se es conocido en la trama que con el Otro y en lo Otro, vivo-concibo-interpreto-produzco-actúo" (SCANNONE, 1976)

Si destacamos que la posibilidad de supervivencia de una organización está dada por su capacidad para diseñarse a sí misma como un sistema social de aprendizaje (Wenger, 1998), indagar creativamente en las brechas nos brinda la posibilidad de observar el flujo abierto, las bifurcaciones y amplificaciones que ofrece la totalidad de la realidad no circunscripta. 


\section{Bibliografía}

ARGYRIS, C. On organizacional learning. 2nd ed. Boston: Blackwell Publishers, 1999.

ARGYRIS, C.; SCHÖN, D. Theory in practice: increasing professional effectiveness. San Francisco: Jossey-Bass Publishers, 1974.

ARGYRIS, C.; SCHÖN, D. Organizational learning: a theory of action perspective. Reading MA: Addison Wesley, 1978.

Ascorra, P. El gerenciamiento de los estados de ánimo: estudio de caso en una organización chilena. Revista Irice. Nueva Época. n.20, p.21-32, nov. 2009.

BALL, S. La micropolítica de la escuela. Hacia una teoría de la organización escolar. Madrid: Paidós/MEC, 1989.

BARDISA RUIZ, T. Teoría y práctica de la micropolítica en las organizaciones escolares. Revista lberoamericana de Educación, España, n.15, 1997.

Bateson, G. A Sacred Unity: Further Steps to an Ecology of Mind. New York: Harper Collins Pub., 1991.

Blasé, J.; Blasé, J. The micropolitical orientation of facilitative school principals and its effects on teachers' sense of empowerment. Journal of Educational Administration, MCB University Press, v.35, n.2, p.138-164, 1997.

BLUMER, H. Symbolic interactionims: perspective and method. Englewoods Cliff, NJ: Prentice-Hall, 1969.

BÖHM, D. Wholeness and the implicate order. Londres: Routledge and Kegan Paul, 1980.

BÖHM, D. On dialogue. España: Kairós, 1994.

BÖHM, D. Unfolding memory. Loveland, CO: Foundation House, 1995.

BRIGGS, J.; PEAT, D. A través del maravilloso espejo del universo. Barcelona: Gedisa, 1999.

CAPRA, F. The Web of life. New York: Anchor Books, 1996.

COHEN, G Amar y permanecer. Basado en el mito de Psiquis y Eros. Buenos Aires: Luz de luna. 2005.

COSTA, L.; PERLO, C.; DE LA RIESTRA, M. R. El diálogo como estrategia para el desarrollo de la mente colectiva en las organizaciones: hacia un modelo de intervención. Familia e Comunidade. Nufac, Pontificia Universidade Católica de San Pablo, São Paulo, Brasil, v.2, n.2, p.11-36, dic. 2005.

DÁVILA YÁNEZ, X; MATURANA, H. La gran oportunidad: fin del liderazgo, en el surgimiento de la gerencia co-inspirativa. In: Entendimiento matriztico organizacional: en el fin del liderazgo y el comienzo de la gerencia co-inspirativa. Santiago de Chile: Instituto Matriztico, 2007. En preparación.

Domato, M. Somos cuerpo. Morada de lo inconcluso. Argentina: Omeba, 2007.

DRUCKER, P (1950) The new society. New York: Harper \& Brothers, 1950.

ESTRAMIANA, J. L. A. (Comp.). Fundamentos sociales del comportamiento humano. Barcelona: UOC, 2003.

FERNÁNDEZ LONGO, E. Ponerse de acuerdo en ponerse de acuerdo. 1997. Disponible en: <www.inicia.org.ar>. Consultado en: 23 feb. 2010.

FLORES, F. Creando organizaciones para el futuro. Santiago de Chile: Dolmen, 1994.

GALTIERI M. Psicología social, modelos de interacción. Estudio preliminar y selección de textos. Buenos Aires: Centro Editor de América Latina, 1992.

López Yánez, J. Hacia una ecología de la organización. Una perspectiva educativa. Madrid: La Muralla, 2005.

LUHMANN, N. La ciencia de la sociedad. Barcelona: Anthropos, 1987.

MATURANA, H. Biología del amor. Sitio Blog y Ciencia. Disponible en: <www.blogyciencia.blogpot.com/2005/10/labiología-del-amor/html>. Consultado em: 2 mayo 2007.

MEAD, G. Espíritu, persona y sociedad. Desde el punto de vista del conductismo social. Buenos Aires: Paidós, 1972.

MORIN, E. Introducción al pensamiento complejo. Barcelona: Gedisa, 1995.

NAJMANOVICH, D. Mirar con otros ojos. Argentina: ED. Biblos, 2008.

Paymal, N. Pedagogía 3000. Guía práctica para docentes, padres y uno mismo. Córdoba: Editorial Brujas, 2008. 
PERLO, C. La organización como producto de la acción individual y colectiva: el aprendizaje organizacional. In: SAGASTIZABAL, M. (Coord.). Aprender y enseñar en contextos complejos. Multiculturalidad, diversidad y fragmentación. Cap.5, Buenos Aires: Noveduc, 2006‥

PERLO, C Aportes del interaccionismo simbólico a las teorías de la organización”. Revista Invenio, Universidad del Centro Latinoamericano-Ucel, Rosario, jun. 2006b.

PERLO, C.; COSTA, L.; DE LA RIESTRA, M. R. Herramientas para la implementación del proceso de diálogo en las organizaciones" (115-128). Revista Invenio, Universidad del Centro Latinoamericano-UCEL, Rosario, año 11, n.21, 2008.

SCANNONE, J. C. Trascendencia, praxis liberadora y lenguaje. In: Teología de la liberación y praxis popular. Salamanca: Sígueme, 1976.

SENGE, P. La quinta disciplina. El arte y la práctica de la organización abierta al aprendizaje. Buenos Aires: Granica, 1992. SERNA ARANGO, J. Somos tiempo. Critica a la simplificación del tiempo de Occidente. Barcelona: Anthopos, 2009.

TERRÉN, E. Las organizaciones educativas como sistemas de comunicación. Un enfoque micropolítico. Revista Iberoamericana de Educación, n.36, p.189-214, 2004.

Toro Araneda, R. Educação biocêntrica. Disponible en: <www.educacaobiocentrica.com.br>. Consultado en: 15 feb. 2010.

WENGER, E. Communities of practice: learning, meaning and identity. Cambridge, UK: Cambridge University Press, 1998. 